\title{
Modeling and Design of an Anti-Vibration Control System
}

\author{
Wu-Sung Yao ${ }^{1}$ \\ ${ }^{1}$ Department of Mechanical and Automation Engineering, National Kaochsiung First University of Science and \\ Technology, Kaohsiung, Taiwan \\ Correspondence: Wu-Sung Yao, Department of Mechanical and Automation Engineering, National Kaochsiung \\ First University of Science and Technology, Kaohsiung, Taiwan. Tel: 886-7-601-1000 ext 7616. E-mail: \\ wsyao@nkfust.edu.tw
}

\author{
Received: January 5, 2014 Accepted: February 4, 2015 Online Published: March 17, 2015 \\ doi:10.5539/apr.v7n2p98 URL: http://dx.doi.org/10.5539/apr.v7n2p98
}

\begin{abstract}
This paper presents an active anti-vibration control system with isolation of low-frequency vibration. A proper dynamic model of the anti-vibration control system is derived for controller design in that the identification scheme for a mathematic model is proposed. The model reference controls of the anti-vibration system with the sky-hook damper control and impedance control are analyzed respectively. An illustrated example of a single-degree-of-freedom anti-vibration system driven by a linear servomotor is given to verify the performance of the proposed control design. Experimental results are presented to validate that the peak resonance value of $0 \mathrm{~dB}$ within a frequency of $10 \mathrm{~Hz}$ can be achieved successively.
\end{abstract}

Keywords: active anti-vibration control, dynamic model, sky-hook damper, impedance control, linear servomotor

\section{Introduction}

\subsection{Introduce the Problem}

Isolating a piece of dainty machines from the vibration source is a practical issue for typical engineering areas. For example, in search of tighter production common difference and substantial resolution places strict specifications. In the plurality of cases, the structure base is always flexible, and easy vibrates having an uncertain signal with broadband harmonic spectrum. With the advancements in manufacturing technologies in modern times, requirements for anti-vibration have grown to become a significant issue. In general, anti-vibration systems can be classified as passive, semi-active, or active. Passive anti-vibration instruments are proverbially employed to hold the equipment in avoiding the base vibration. Nevertheless, traditional passive anti-vibrations acquire an intrinsic trade-off between weak high-frequency isolation and vibration amplification at the resonant frequency. Semi-active anti-vibration systems can be operated within the same framework as passive ones but are incorporated with an adjustable damper or spring, which allows for variation in the damping coefficients. Early semi-active dampers are mechanically adjustable by a bypass valve. For example, by changing the liquid pressure, the damping coefficient can be modified to achieve different specifications (Marjoram, 1985). The semi-active anti-vibration system significantly increases the capability in dealing with uncertainties; however, it is difficult to against low frequency vibrations. The active anti-vibration system, on the other hand, can achieve different goals in various applications and has become increasingly popular in academic research and industry. In the active anti-vibration, extra energy is actively generated by the controlled actuators to reduce vibrations, such as the tuned mass dampers that are atop Taipei 101 (Tuned mass damper atop the Taipei 101, ND) and applied to offshore platforms to counter low frequency vibrations (Zhang, Ma, \& Zhao, 2010). Various anti-vibration control strategies have been discussed, including feedforward and feedback control concepts for systems under periodic and/or random vibrations. For examples, HWL Scientifice Instrument GmbH (ND) and Lee, Chen, Chung, and Wang (2006) utilized both feedforward and feedback control techniques to enhance the anti-vibration performance. In Yu, Naganathan, and Dukkipati (2001), an independent two-channel controller based on velocity feedback was employed to improve the isolation from the base vibration of a mounted rigid-equipment structure. Theoretical and experimental investigations of active anti-vibration systems were also presented in Marjoram (1985) and Beard, Schubert, and Von Flotow (1994). The conventional approach, which adopts an equivalent mass with a damper and spring connected to the base, often leads to the trade-off of anti-vibration performance between high and low frequencies. For coping with this shortcoming, Karnopp, Crosby, and Harwood (1974) introduced a sky-hook damper, which significantly ameliorated the effect of this trade-off design. However, due to restrictions in physics, anti-vibration 
systems with a sky-hook damper are difficult to be realized. In the author's previous study (Yao, Hsueh, \& Tsai, 2013), the isolation of low-frequency vibration has been studied. Also, a model reference control of the anti-vibration system with a sky-hook damper and impedance control was analyzed. However, to achieve a desired anti-vibration performance, an accurate dynamic model of the controlled system is required for controller design in that the identification for a mathematic model is the key point. In this paper, based on the achievement of Yao, Hsueh, and Tsai (2013), an on-line tuning identification based on the recursive least-square errors is proposed, and model reference controls of the anti-vibration system with the sky-hook damper control and impedance control are analyzed. An illustrated example of a single-degree-of-freedom anti-vibration system driven by a tubular linear servomotor is given to verify the performance of the proposed control design. Experimental results are given to illustrate the performance of the proposed identification technique and anti-vibration control.

\section{Analysis of Anti-Vibration System}

\subsection{Passive Anti-Vibration for Base-Excited System}

Figure 1 shows a SDOF (single degree-of-freedom) passive anti-vibration system, where $m$ is the equivalent mass, $k$ is the spring coefficient, and $c$ is the damping coefficient. $x$ denotes the displacement of the controlled object $m$, and $x_{b}$ is the displacement of the base structure. The dynamic equation of the controlled system in Figure 1 is given as

$$
m \ddot{x}+c\left(\dot{x}-\dot{x}_{b}\right)+k\left(x-x_{b}\right)=0
$$

Employing the Laplace transform yields

$$
\frac{X}{X_{b}}=\frac{c s+k}{m s^{2}+c s+k}=\frac{c s / m+k / m}{s^{2}+c s / m+k / m}
$$

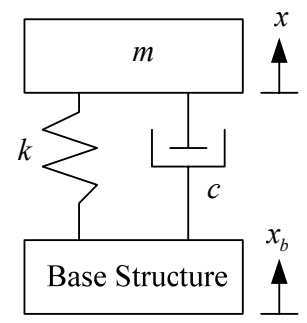

Figure 1. SDOF base-excited passive anti-vibration system

Observing (2), the mathematics modelling of the passive anti-vibration system can be equivalently found as a unity feedback control system with PI (Proportional and Integral) controller can be given, where the controlled plant can be represented by $1 / \mathrm{s}$, as shown in Figure 2 . Therefore, $\mathrm{c} / \mathrm{m}$ and $\mathrm{k} / \mathrm{m}$ are equivalently the proportional and integral gains of the PI controller respectively. Based on the conventional PI control design, the response speed of the system increases with $\mathrm{c} / \mathrm{m}$ of the proportional gain increasing, the overshoot of the closed-loop system increases, and the steady-state error decreases. However when $c / m$ is large enough, and the closed-loop system becomes unstable. With $c / m$ fixed and $\mathrm{k} / \mathrm{m}$ of the integral gain increasing, the overshoot tends to be smaller, but the speed of response tends to be slower. It is known that the PI controller does not offer a good disturbance rejection performance compared to other types of controllers.

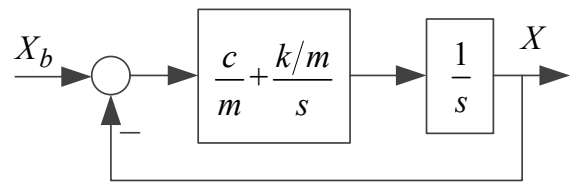

Figure 2. A unity feedback control system with PI controller

To quantify the control performance of an anti-vibration system, the transmissibility is an important index that can be defined as a displacement, velocity, or acceleration ratio between the base structure and the controlled object $m$. The transmissibility $T_{p}(s)$ quantified by the displacement is denoted as 


$$
T_{p}(\omega)=\left|\frac{X(j \omega)}{X_{b}(j \omega)}\right|=\sqrt{\frac{1+4 \zeta^{2}\left(\omega / \omega_{n}\right)^{2}}{1+\left(4 \zeta^{2}-2\right)\left(\omega / \omega_{n}\right)^{2}+\left(\omega / \omega_{n}\right)^{4}}}
$$

where $\omega_{n}=\sqrt{k / m}$ is the natural frequency and $\zeta=c / 2 \sqrt{m k}$ is the damping ratio. Note that the transmissibility of (3) depends on the natural frequency and the damping ratio of the system. A simulated example of $m=5, c=2 \sim 3$, and $k=8$ is adopted. The transmissibility being a function of $\omega / \omega_{n}$ can be acquired, leading to Figure 3 for various damping ratios. For lower damping ratios, the resonant transmissibility at $\omega=\omega_{n}$ is relatively substantial, while the transmissibility is pretty lower under frequencies over the resonant point. We now have the resonant transmissibility of (3) is $\sqrt{\left(1+4 \zeta^{2}\right) / 4 \zeta^{2}}$ at $\omega=\omega_{n}$. The opposite is true for relatively high damping ratios. If a low damping ratio is determined, superior high frequency isolation can be obtained with poor resonant frequency control. Nonetheless, to increase the damping ratio, the higher frequency isolation for resonance control can be to trade off.

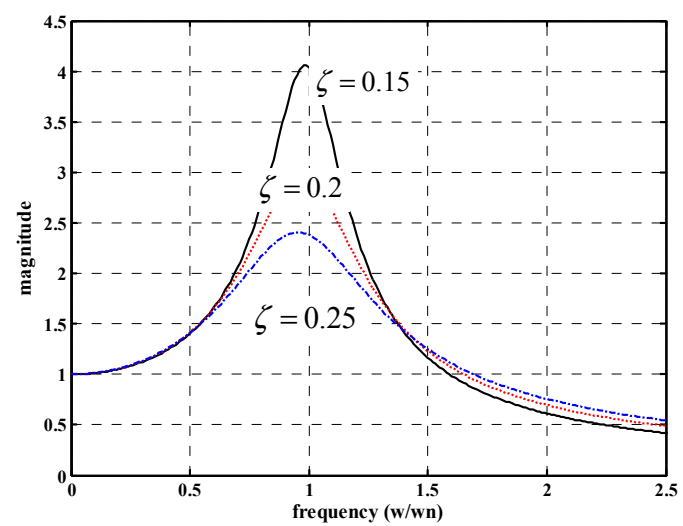

Figure 3. Transmissibility of passive anti-vibration system in Figure 1

\subsection{Sky-Hook Damper}

In order to accomplish a suitable tradeoff between resonance control as well as higher frequency isolation, a sky-hook damper is obtained in the study as shown in Figure 4. The dynamic equation of the sky-hook damper model is given by

$$
m \ddot{x}+c_{h} \dot{x}+k\left(x-x_{b}\right)=0
$$

and hence

$$
\frac{X}{X_{b}}=\frac{k}{m s^{2}+c_{h} s+k}=\frac{k / m}{s^{2}+c_{h} s / m+k / m}
$$

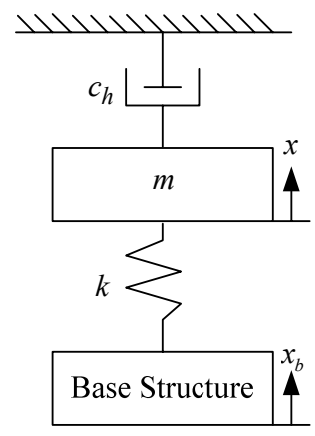

Figure 4. Ideal sky-hook configuration 
Observing (5), the mathematics modelling of Figure 4 can be equivalently found as a feedback control system with PDF (Pseudo Derivative Feedback) controller, where the controlled plant can be represented by $1 / s$, as shown in Figure 5. Therefore, $c_{h} / m$ and $k / m$ are equivalently the proportional and integral gains of the PDF controller respectively. In this scheme, proportional gain $c_{h} / m$ is acting only on the output $x$. The transmissibility $T_{s}(\omega)$ of the ideal sky-hook damper structure is then given by

$$
T_{s}(\omega)=\frac{1}{\sqrt{1+\left(4 \zeta_{h}^{2}-2\right)\left(\omega / \omega_{n}\right)^{2}+\left(\omega / \omega_{n}\right)^{4}}}
$$

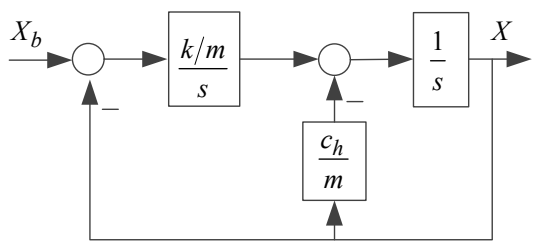

Figure 5. A feedback control system with PDF controller

where the damping ratio is $\zeta_{h}=c_{h} / 2 \sqrt{m k}$ The transmissibility for various $\zeta_{h}$ is shown in Figure 6. The resonant transmissibility of (6) is $1 / 2 \zeta_{h}$ at $\omega=\omega_{n}$. With regard to the passive event, the resonant transmissibility will likely be decreased for raising skyhook damping ratio. For $\zeta_{h}>0.707$, the resonant frequency may even be isolated. Consequently, we can avoid the tradeoff involving passive dampers. The passive suspension (i.e., both the damper and the spring) could be replaced by an active force generator to provide the damping force. This is certainly accomplished through a hydraulic actuator; but, the resulting system is quite complex and requires certain power. An additional approach is to try using semi-active dampers to realize skyhook damping, which provide for adjusting the damping coefficient. In this paper, a linear servomotor is given to generate a control force for the active anti-vibration control system. In practical, the parameters of the controlled plant are always difficult to obtain such that the control performance of the anti-vibration control will be reduced due to the parameters' variation. Therefore, an on-line tuning identification is also proposed, and a model reference control of the anti-vibration system is analyzed.

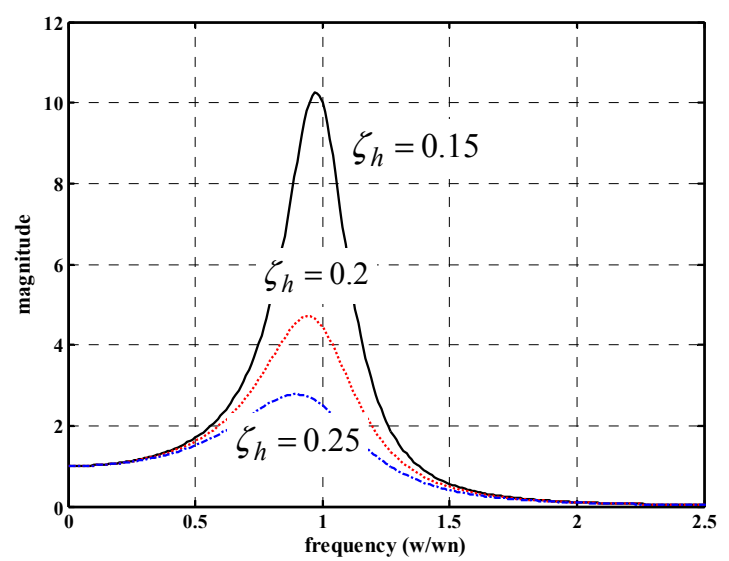

Figure 6. Transmissibility of ideal sky-hook damper in Figure 4

\section{Parameter Estimation of Controlled Plant}

Linear least squares can be used to transfer a mathematical model to data in cases where the data point can be linearly expressed in terms of the unknown model parameters. The resulting fitted model can be used to summarize the data, to predict unobserved values of the system. Assume that the output of the linear model can be given by the linear parameterized expression, i.e.,

$$
\mathbf{Y}=\Phi \Theta
$$


$\mathbf{Y} \in \mathbb{R}^{m \times 1}$ is the output matrix of the system, i.e., $\mathbf{Y}=\left[\begin{array}{llll}y_{1} & y_{2} & \cdots & y_{m}\end{array}\right]^{T} ; \boldsymbol{\Theta} \in \mathbb{R}^{n \times 1}$ are the parameters to be estimated, i.e., $\boldsymbol{\Theta}=\left[\begin{array}{llll}\theta_{1} & \theta_{2} & \cdots & \theta_{n}\end{array}\right]^{T}$; and $\boldsymbol{\Phi} \in \mathbb{R}^{m \times n}$ is the information matrix from input to output of the system, i.e.,

$$
\boldsymbol{\Phi}=\left[\begin{array}{ccc}
\varphi_{1}\left(u_{1}\right) & \cdots & \varphi_{n}\left(u_{1}\right) \\
\vdots & \vdots & \vdots \\
\varphi_{1}\left(u_{m}\right) & \cdots & \varphi_{n}\left(u_{m}\right)
\end{array}\right]
$$

where $u_{1}, u_{2}, \ldots$, and $u_{m}$ are the system inputs; and, $\varphi_{1}, \varphi_{2}, \ldots$, and $\varphi_{n}$ are functions of $u_{1}, u_{2}, \ldots$, and $u_{m}$, respectively.

Assume that $\boldsymbol{\Theta}=\left(\boldsymbol{\Phi}^{T} \boldsymbol{\Phi}\right)^{-1} \boldsymbol{\Phi}^{T} \mathbf{Y}$ can be obtained. Then, based on (7) and (8), we have

$$
\Theta_{n}=\boldsymbol{\Gamma}_{n}^{-1} \sum_{k=1}^{n} \boldsymbol{\Phi}^{T}(k) \mathbf{Y}(k)
$$

where

$$
\begin{aligned}
& \boldsymbol{\Gamma}_{n}=\sum_{k=1}^{n} \boldsymbol{\Phi}^{T}(k) \boldsymbol{\Phi}(k) \text { and } \boldsymbol{\Theta}_{n+1}=\boldsymbol{\Gamma}_{n+1}^{-1} \sum_{k=1}^{n+1} \boldsymbol{\Phi}^{T}(k) \mathbf{Y}(k) \text {. Therefore, we obtain } \\
& \qquad \sum_{k=1}^{n+1} \boldsymbol{\Phi}^{T}(k) \mathbf{Y}(k) \\
& =\boldsymbol{\Gamma}_{n+1} \Theta_{n}+\boldsymbol{\Phi}^{T}(n+1)\left[\mathbf{Y}(n+1)-\boldsymbol{\Phi}(n+1) \boldsymbol{\Theta}_{n}\right]
\end{aligned}
$$

Then,

$$
\begin{aligned}
& \boldsymbol{\Theta}_{n+1}=\boldsymbol{\Theta}_{n}+\boldsymbol{\Gamma}_{n+1}^{-1} \boldsymbol{\Phi}^{T}(n+1)\left[\mathbf{Y}(n+1)-\boldsymbol{\Phi}(n+1) \boldsymbol{\Theta}_{n}\right] \\
& \boldsymbol{\Gamma}_{n+1}=\boldsymbol{\Gamma}_{n}+\boldsymbol{\Phi}^{T}(n+1) \boldsymbol{\Phi}(n+1) \\
& \boldsymbol{\Gamma}_{n+1}^{-1}=\boldsymbol{\Gamma}_{n}^{-1}- \\
& \boldsymbol{\Gamma}_{n}^{-1} \boldsymbol{\Phi}^{T}(n+1)\left[\mathbf{I}+\boldsymbol{\Phi}(n+1) \boldsymbol{\Gamma}_{n}^{-1} \boldsymbol{\Phi}^{T}(n+1)\right]^{-1} \boldsymbol{\Phi}(n+1) \boldsymbol{\Gamma}_{n}^{-1}
\end{aligned}
$$

Define $\Lambda:=\Gamma^{-1}$; then, the RLS of the system parameter is given by

$$
\Theta_{n}=\Theta_{n-1}+\Lambda_{n} \Phi^{T}(n) \mathbf{E}(n)
$$

where $\mathbf{E}(n)$ is the parameter estimated error and can be described as

$$
\mathbf{E}(n):=\mathbf{Y}(n)-\boldsymbol{\Phi}^{T}(n) \boldsymbol{\Theta}_{n-1}
$$

Then, we have

$$
\begin{aligned}
& \boldsymbol{\Lambda}_{n}=\boldsymbol{\Lambda}_{n-1}- \\
& \boldsymbol{\Lambda}_{n-1} \boldsymbol{\Phi}^{T}(n)\left[\mathbf{I}+\boldsymbol{\Phi}(n) \boldsymbol{\Lambda}_{n-1} \boldsymbol{\Phi}^{T}(n)\right]^{-1} \boldsymbol{\Phi}(n) \boldsymbol{\Lambda}_{n-1}
\end{aligned}
$$

Based on (16), the parameters of the controlled plant can be determined for further controller design.

\section{Illustrated Example and Parameter Estimation}

An illustrated example of an anti-vibration control system was on purpose developed to verify the control performance of the proposed control scheme. Figure 7 exhibits the experimental setup, where the linear electric motor provides an active force control. A ball-screw system driven by a rotary electric motor can be used to produce disturbances. The intention of the control design is to minimize oscillations of the controlled target, which is marked by the dashed-line box in Figure 7. In fact, a control system modeling of the experimental setup depicted in Figure 7 can be detailed as shown in Figure 8, where 


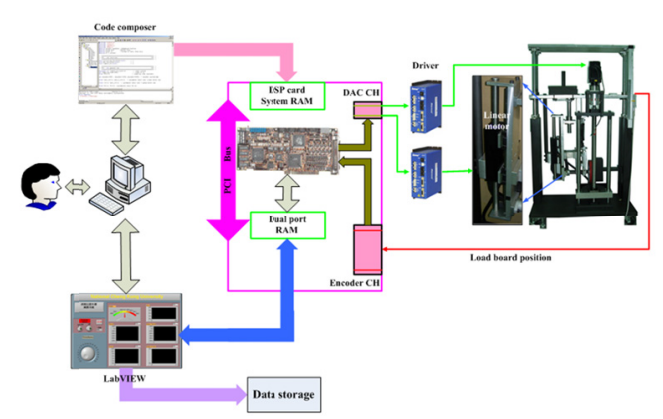

Figure 7. Experimental setup

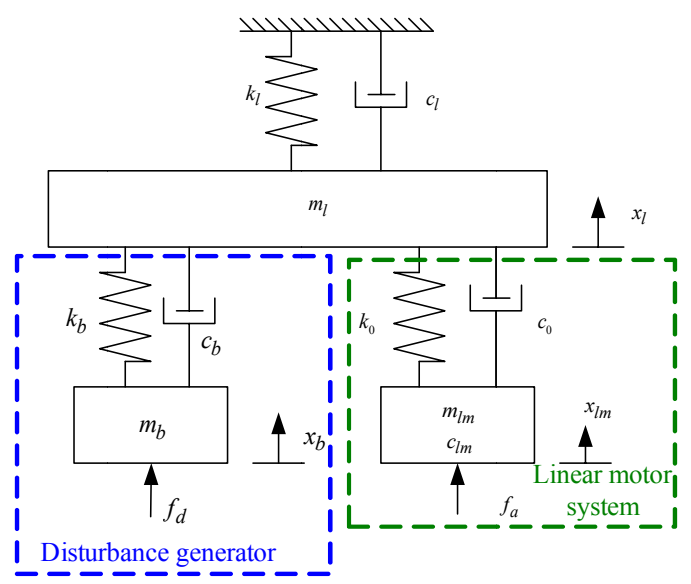

Figure 8. Control system modeling of experiment setup of Figure 7

$k_{b}$ : spring coefficient of ball-screw system

$m_{b}$ : mass of ball-screw system

$c_{b}$ : damping coefficient of ball-screw system

$x_{b}$ : displacement of ball-screw system

$f_{d}$ : force generated by ball-screw system

$m_{l m}:$ mass of linear motor

$c_{l m}$ : damping coefficient of linear motor

$f_{a}$ : force generated by linear motor

$x_{l m}:$ displacement of linear motor

$k_{0}$ : spring coefficient of mechanical coupling between linear motor and loader

$c_{0}$ : damping coefficient of mechanical coupling between linear motor and loader

$m_{l}:$ mass of loader

$k_{l}$ : spring coefficient of passive component

$c_{l}$ : damping coefficient of passive component

$x_{l}$ : displacement of loader.

Then, we have the control block diagram of the experimental system as shown in Figure 9.

To design an anti-vibration controller, the controlled system in Figure 9 can be simplified to a second-order dynamic system as depicted in Figure 10. $k$ and $c$ denote the equivalent stiffness and damping effects of the passive anti-vibration elements, respectively. $m_{l}$ and $m_{b}$ are the equivalent loading of the mechanical 
elements and the equivalent mass of the disturbance generating device, respectively. Based on Figure 10, the dynamic equation of the proposed anti-vibration system is given as (17), and its Laplace transform can be given (18). Then, the block diagram of the controlled system is shown in Figure 11.

$$
\begin{aligned}
& \left\{\begin{array}{l}
m_{b} \ddot{x}_{b}+c\left(\dot{x}_{b}-\dot{x}_{l}\right)+k\left(x_{b}-x_{l}\right)=f_{d} \\
m_{l} \ddot{x}_{l}+c\left(\dot{x}_{l}-\dot{x}_{b}\right)+k\left(x_{l}-x_{b}\right)=f_{a}
\end{array}\right. \\
& {\left[\begin{array}{l}
X_{l} \\
X_{b}
\end{array}\right]=\frac{1}{\Delta}\left[\begin{array}{cc}
m_{b} s^{2}+c s+k & c s+k \\
c s+k & m_{l} s^{2}+c s+k
\end{array}\right]\left[\begin{array}{l}
F_{a} \\
F_{d}
\end{array}\right]}
\end{aligned}
$$

where $\Delta=m_{b} m_{l} s^{4}+\left(m_{b}+m_{l}\right) c s^{3}+\left(m_{b}+m_{l}\right) k s^{2}$.

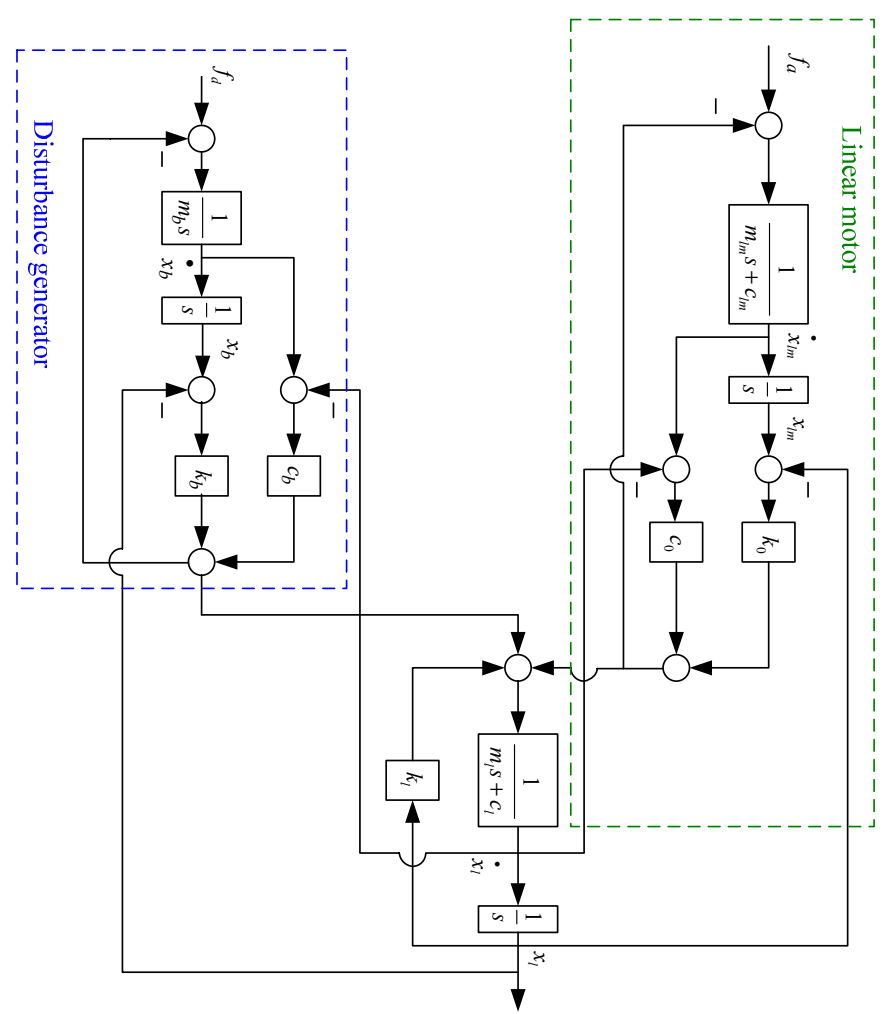

Figure 9. Control block diagram of controlled system

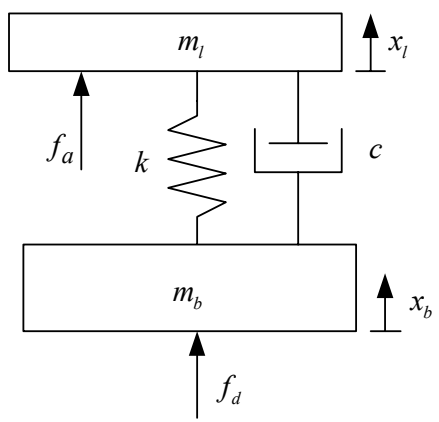

Figure 10. Simplified model of Figure 9 


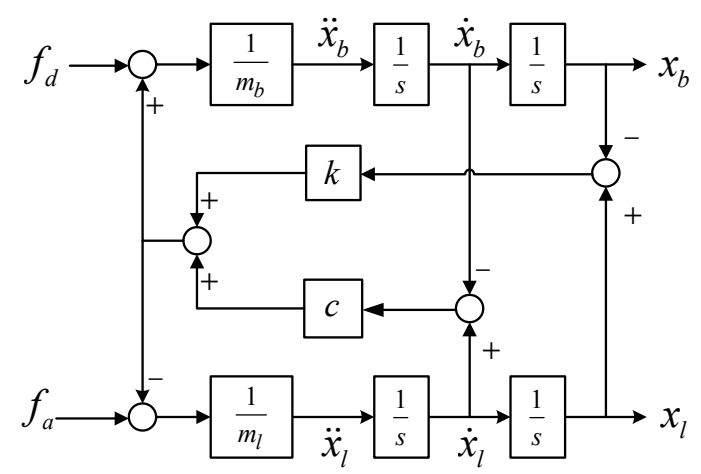

Figure 11. Block diagram of proposed anti-vibration system.

For the requirement of $X_{l}=0$ with $F_{d}$, the control force of the tubular linear motor should be given as $F_{a}=-\frac{c s+k}{m_{b} s^{2}+c s+k} F_{d}$. In practice, the information of $F_{d}$ is difficult to obtain. Therefore, in this paper, an appropriate control scheme is adapted to reduce the effect of the disturbance $F_{d}$. To estimate the parameters of $m_{l}, k$, and $c$ under $F_{d}=0$, the dynamics of the system is given as

$$
\frac{X_{l}}{F_{a}}=\frac{\left(m_{b} s^{2}+c s+k\right)}{m_{b} m_{l} s^{4}+\left(m_{b}+m_{l}\right) c s^{3}+\left(m_{b}+m_{l}\right) k s^{2}}
$$

Assuming $m_{b} \gg m_{l}$ in this case, (19) can be simplified as

$$
\frac{X_{l}}{F_{a}}=\frac{1}{m_{l} s^{2}+c s+k}
$$

The parameters of the controlled system can be estimated by the proposed scheme in Section III. Then, we have

$$
\begin{aligned}
& m_{l} \frac{x_{l}[n]-2 x_{l}[n-1]+x_{l}[n-2]}{T^{2}} \\
& +c \frac{x_{l}[n]-x_{l}[n-1]}{T}+k x_{l}[n]=f_{a}[n] \\
& x_{l}[n]=\frac{2 m_{l}+c T}{m_{l}+c T+k T^{2}} x_{l}[n-1] \\
& +\frac{-m_{l}}{m_{l}+c T+k T^{2}} x_{l}[n-2]+\frac{T^{2}}{m_{l}+c T+k T^{2}} f_{a}[n]
\end{aligned}
$$

where $T$ is the sampling time, and $n$ is the sampling number. Then, $\mathbf{Y}=\boldsymbol{\Phi} \Theta$ can be obtained, i.e.,

$$
\begin{gathered}
\mathbf{Y}=x_{l}[n] \\
\mathbf{\Phi}=\left[\begin{array}{lll}
\frac{2 m_{l}+c T}{m_{l}+c T+k T^{2}} & \frac{-m_{l}}{m_{l}+c T+k T^{2}} & \frac{T^{2}}{m_{l}+c T+k T^{2}}
\end{array}\right] \\
\boldsymbol{\Theta}=\left[\begin{array}{lll}
x_{l}[n-1] & x_{l}[n-2] & \left.f_{a}[n]\right]^{T}
\end{array}\right.
\end{gathered}
$$

In this case, based on (16), the initial condition of $\Phi(0)=0$ and $\Lambda(0)=5000$ is given. To verify the accuracy of the proposed estimator, three repetitions for each estimation are done with varying masses of $10 \mathrm{~kg}$ and $30 \mathrm{~kg}$. Figures 12 and 13 show that the parameters can be accurately projected by the proposed estimator, where $m_{l}=30 \mathrm{~kg}, k=30000 \mathrm{~N} / \mathrm{m}$, and $c=250 \mathrm{Ns} / \mathrm{m}$ are determined in the case of Figure 10. In actuality, $m_{b}=400 \mathrm{~kg}$ can be measured in this illustrated example. Based on the pre-identified parameters (i.e., $m_{l}=30 \mathrm{~kg}, k=30000 \mathrm{~N} / \mathrm{m}$, and $\left.c=250 \mathrm{Ns} / \mathrm{m}\right)$, the frequency response of the mathematic modeling (18) in Figure 11 has been verified by the experimental measurement as shown in Figure 14. 


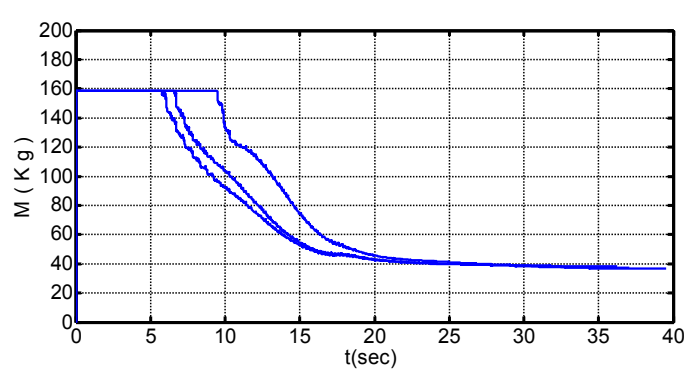

(a) equivalent mass $m_{l}$

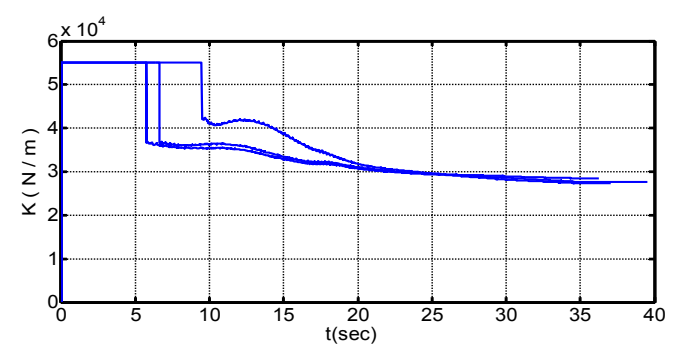

(c) equivalent spring coefficient $k$

Figure 12. Estimated results with extra $10 \mathrm{~kg}$ loading

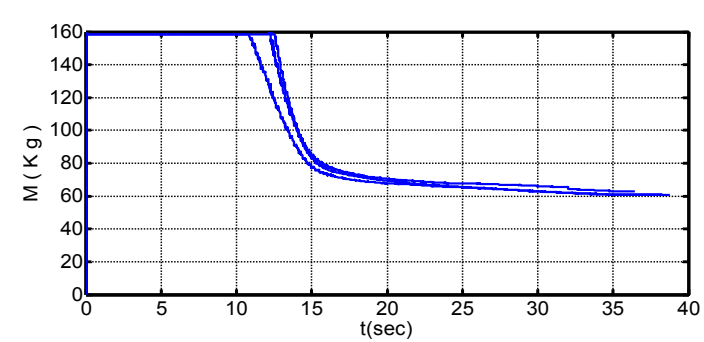

(a) equivalent mass $m_{l}$

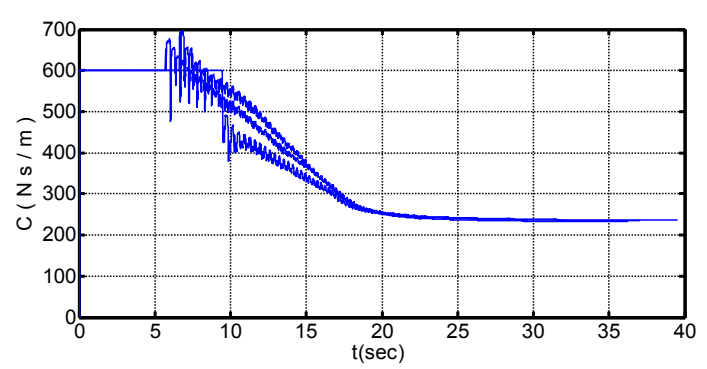

(b) equivalent damping coefficient $c$

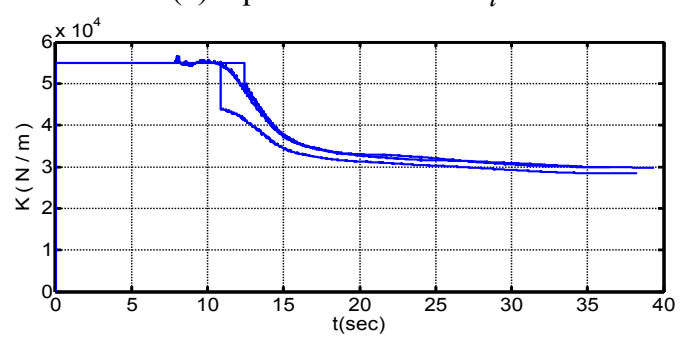

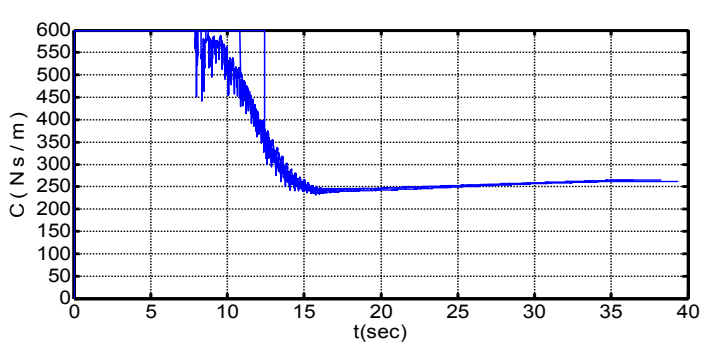

(b) equivalent damping coefficient $c$

(c) equivalent spring coefficient $k$

Figure 13. Estimated results with extra $30 \mathrm{~kg}$ loading

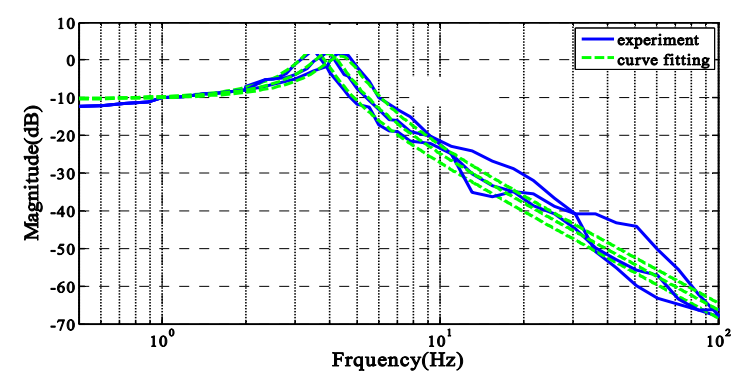

Figure 14. Frequency responses of controlled system with extra mass of $10 \mathrm{~kg} \sim 30 \mathrm{~kg}$. 


\section{Analysis of Active Anti-Vibration Control System}

The proposed anti-vibration system can be modeled as shown in Figure 11, where the transmissibility is given as

$$
T_{p}(\omega)=\sqrt{\frac{\left(2 \zeta_{p} \omega\right)^{2}+\omega_{n}^{2}}{\left(2 \zeta_{p} \omega\right)^{2}+\omega_{n}^{2}\left(1-\omega^{2} / \omega_{n}^{2}\right)^{2}}}
$$

where $\omega_{n}=\sqrt{k / m_{l}}$ and $\zeta_{p}=c /\left(2 \sqrt{m_{l} k}\right)$.

From (26), the resonant transmissibility can be obtained as $\left(1+4 \zeta_{p}^{2}\right) / 4 \zeta_{p}^{2}$ at $\omega=\omega_{n}$, while the transmissibility at frequencies above the resonant peak is quite low. In this paper, the active anti-vibration control system can be described with a feedback controller $H(s)$ as shown in Figure 15, where $H(s)$ performs in the role of a sky-hook damper and an impendence controller. The transmissibility of Figure 15 is given as

$$
\hat{T}_{p}(\omega)=\left|\frac{j \omega c+k}{-m_{l} \omega^{2}+j \omega c+k-H(j \omega)}\right| .
$$

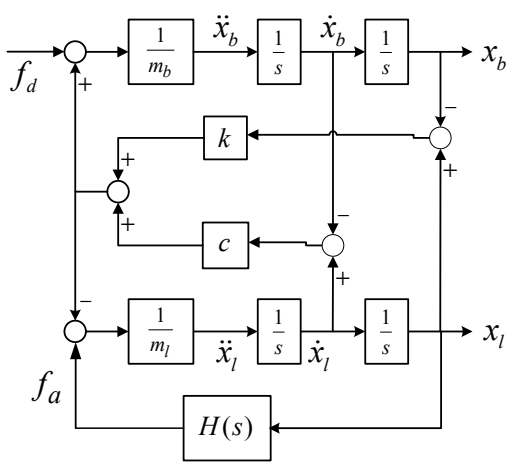

Figure 15. Block diagram of the active anti-vibration control system with feedback controller $H(s)$

Moreover, we have

$$
\frac{X_{l}}{F_{d}}=\frac{c s+k}{\Delta_{H}(s)}
$$

where

$$
\begin{aligned}
& \Delta_{H}(s)=m_{b} m_{l} s^{4}+\left(m_{b}+m_{l}\right) c s^{3}+\left(m_{b}+m_{l}\right) k s^{2} \\
& -\left(m_{b} s^{2}+c s+k\right) H(s)
\end{aligned}
$$

$H(s)$ should be determined such that

$$
\left|\frac{\Delta(j \omega)}{\Delta_{H}(j \omega)}\right|<1
$$

Then, the vibration can be effectively reduced by using the active-vibration control system in Figure 13 . The purpose of the sky-hook damper control is to add the virtual damper into the anti-vibration system by $H(s)=-c_{h} s$. Then, the linear motor force can be determined by $F_{a}=-c_{h} \dot{x}_{l}$. From Figure 15 and (28), the transmissibility of the active anti-vibration with sky-hook damper control is defined as

$$
T_{s}(\omega)=\sqrt{\frac{\left(2 \zeta_{p} \omega\right)^{2}+\omega_{n}^{2}}{4 \zeta_{p}{ }^{2} \omega^{2}+\omega_{n}^{2}\left(1-\omega^{2} / \omega_{n}^{2}\right)^{2}}}
$$

where $\omega_{n}=\sqrt{k / m_{l}}$ and $\zeta_{p}=\left(c+c_{h}\right) /\left(2 \sqrt{m_{l} k}\right)$. 
From (29), the resonant transmissibility with the virtual damper can be obtained as $\left(4 \zeta_{p}^{2}+1\right) / 4\left(\zeta_{p}+\zeta_{h}\right)^{2}$ at $\omega=\omega_{n}$. Because $c_{h}>0$, the resonant transmissibility of (29) is always smaller than that of (26). Therefore, the sky-hook damper control can improve the system damper to reduce system resonance.

In the impedance control, the motion of the active component is used to provide a virtual impedance to improve the performance of the anti-vibration system, and we have $H(s)=-m_{v} s^{2}-c_{v} s$, where $m_{v}$ and $c_{v}$ are the target impedance parameters of the mass and the viscosity. Therefore, we have then

$$
f_{a}=-m_{v} \ddot{x}_{l}-c_{v} \dot{x}_{l}
$$

Then, the transmissibility of the active anti-vibration with impedance control is given as

$$
T_{v}(\omega)=\sqrt{\frac{\omega_{n}^{2}+\left(2 \zeta_{d} \omega\right)^{2}}{\left(2 \zeta_{d} \omega\right)^{2}+\omega_{n}^{2}\left(1-\omega^{2} / \omega_{n}^{2}\right)^{2}}}
$$

where $\omega_{n}=\sqrt{k /\left(m_{l}+m_{v}\right)}$, and $\zeta_{d}=\left(c+c_{v}\right) / 2 \sqrt{\left(m_{l}+m_{v}\right) k}$.

Observing (31), the resonant transmissibility at $\omega=\omega_{n}$ can be obtained as $\left(4 \zeta_{d}^{2}+1\right) / 4 \zeta_{d}^{2}$. Therefore, a required anti-vibration performance can be achieved by the two designed parameters $m_{v}$ and $c_{v}$.

\section{Experiment Results}

The actual proposed control scheme is realized by a digital signal processor, where the control algorithms are performed along with the TI TMS320C30 floating-point processor, and then the sampling time period is given as $0.001 \mathrm{sec}$. In this paper, a low-acceleration estimator (LAE) is adopted to estimate the acceleration and velocity using encoder position data (Lee \& Song, 2001). The block diagram of LAE is shown in Figure 16, where $K_{1}$ and $K_{2}$ are designed parameters. $r$ is the input position signal, while $\hat{r}, \hat{v}$, and $\hat{a}$ are the estimated position, velocity, and acceleration, respectively. In this case, the bandwidth is given as $30 \mathrm{~Hz}$; then, $K_{1}=35494$ and $K_{2}=226$ can be calculated.

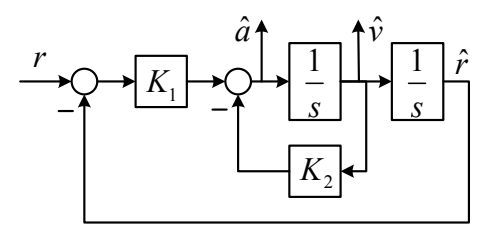

Figure 16. Block diagram of LAE

For sky-hook damper control in Figure 15, the control input $f_{a}=-c_{h} \dot{x}_{l}$ and the velocity $\dot{x}_{l}$ can be calculated by LAE. Therefore, to synthesize the control input and LAE, we have

$$
H_{h}(s)=-c_{h} \frac{35494 s}{s^{2}+266 s+35494}
$$

The root locus plot of Figure 15 with (32) is shown in Figure 17. As $c_{h}$ increases, the poles of $H_{h}(s)$ approach the imaginary axis, which results in system instability. Therefore, for the required stability, the number of $H_{h}(s)$ poles should be at least five times that of the controlled plant in Figure 11 by designing a $c_{h}$ of (32). In the case with impedance control, the control input $f_{a}=-m_{v} \ddot{x}_{l}-c_{v} \dot{x}_{l}$, the velocity $\dot{x}_{l}$, and acceleration $\ddot{x}_{l}$ can be calculated from LAE. Then, we obtain

$$
H_{i m}(s)=-c_{v} \frac{35494 s}{s^{2}+266 s+35494}-m_{v} \frac{35494 s^{2}}{s^{2}+266 s+35494}
$$




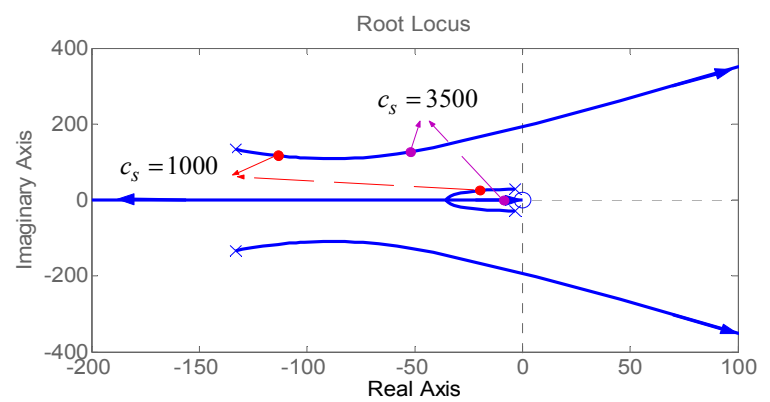

Figure 17. Root locus plot of Figure 14 with (32)

Varying $m_{v}$ and $c_{v}$, the root locus plot of Figure 15 with (33) can be illustrated as in Figure 18. The dashed-line shows the result with $m_{v}=0$, while solid-line shows the trend with $m_{v}$ varying when the value of $c_{v}$ is fixed at 1000 and 3500 , respectively. In a similar consequence to the sky-hook damper control case of (32), the $H_{i m}(s)$ poles tend to gravitate towards the imaginary axis when $c_{v}$ increases.

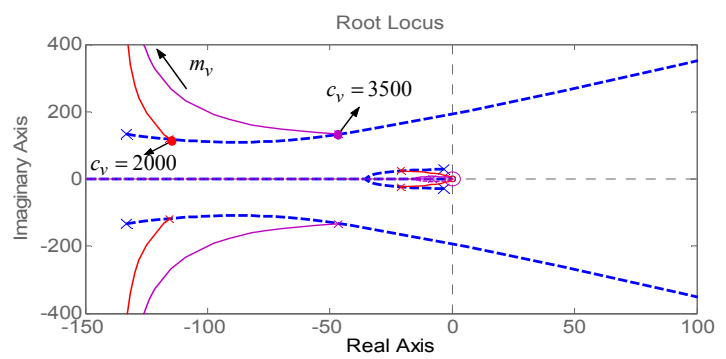

Figure 18. Root locus plot of Figure 14 with (33)

Actively avoiding vibrations within the frequency of $10 \mathrm{~Hz}$ is the goal of the sky-hook damper and impedance control incorporated in the anti-vibration system. An external disturbance force $f_{d}$ is generated by a servo motor with $2 \mathrm{~mm}$ amplitude with the frequency from $1 \mathrm{~Hz}$ to $10 \mathrm{~Hz}$. Thus, the transmissibility in the passive anti-vibration system of Figure 10 with $f_{a}=0$ is shown in Figs 19. The functionality of the anti-vibration is unapparent at less than $6 \mathrm{~Hz}$, and the peak of resonance is about $11 \mathrm{~dB}$ at $4.5 \mathrm{~Hz}$. Further, the transmissibility of the active anti-vibration system with sky-hook damper with (32) are shown in Figure 20, where $c_{v}=300$ and $c_{v}=1200$ are given. The peak of the resonance at about $4.5 \mathrm{~Hz}$ can be clearly restrained to $0 \mathrm{~dB}$.

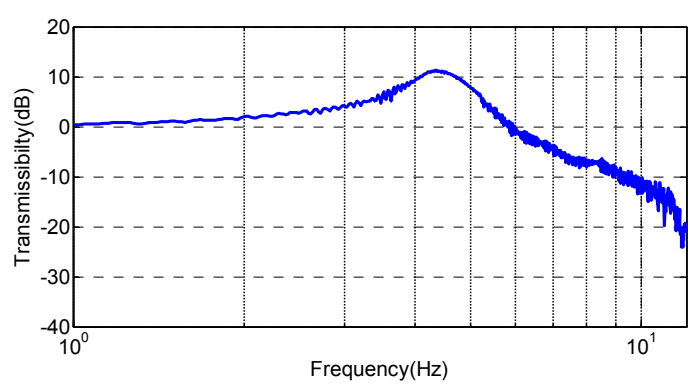

Figure 19. Transmissibility of Figure 10 with $f_{a}=0$

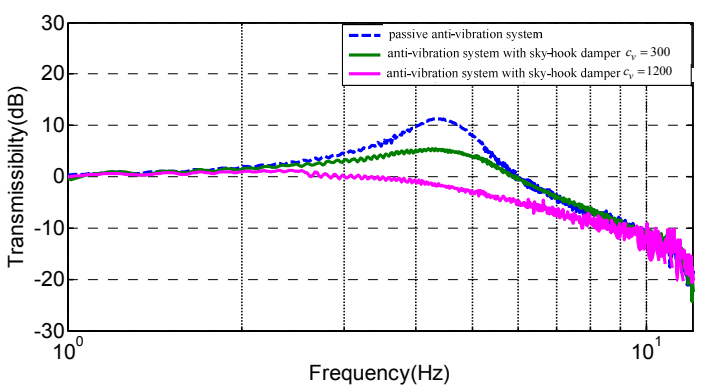

Figure 20. Transmissibility of Figure 12 using sky-hook damper

The impedance control is then implemented into the anti-vibration system; the natural frequency $\omega_{n}$ and damping ratio $\zeta_{d}$ of (31) can be modulated through setting the parameters $m_{v}$ and $c_{v}$. For this experiment, the damping ratio $\zeta_{d}$ is set to 0.707 , and $c_{v}=1200$ can be determined. Subsequently, the trend of the transmissibility can be shown in Figure 21, where $m_{v}$ is set at 0 and 30. From the result, the natural frequency of the system can be reduced while $m_{v}$ increases, but the damping ratio is decreased, which leads to the peak of the resonance becoming more obvious. In order to design an acceptable performance of the active anti-vibration system, it is better to adjust $m_{v}$ and $c_{v}$. 


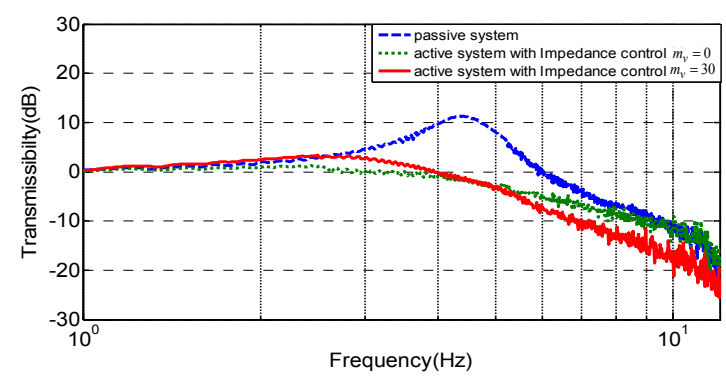

Figure 21. Transmissibility of Figure 12 using impedance control

Figure 22 shows the comparison of the passive system, sky-hook damper control, and impedance control cases. The results are discussed in three frequency ranges: $1 \sim 2.5 \mathrm{~Hz} ; 2.5 \sim 7 \mathrm{~Hz}$; and $7 \sim 10 \mathrm{~Hz}$. First, the anti-vibration ability is unapparent between $1 \mathrm{~Hz}$ to $2.5 \mathrm{~Hz}$ in the passive system and sky-hook damper $\left(c_{S}=1200\right)$ case. However, the vibration can be reduced to the $1 \mathrm{~Hz}$ to $2.5 \mathrm{~Hz}$ range in the anti-vibration with impedance control, while $m_{v}=60$ and $c_{v}=3000$. Second, resonance occurs in the passive system when the frequency of the external vibration is between $2.5-7 \mathrm{~Hz}$. However, the peak of the resonance can be reduced to $0 \mathrm{~dB}$ in the sky-hook damper control and $-5 \mathrm{~dB}$ with the impedance control. Finally, the vibration can be decreased to lower than $-10 \mathrm{~dB}$ between $7 \mathrm{~Hz}$ and $10 \mathrm{~Hz}$ in the passive system and sky-hook damper control. Additionally, it is almost decreased to $-15 \mathrm{~dB}$ in the impedance control within the $7 \sim 10 \mathrm{~Hz}$ range. However, the vibrations seem to occur between $7 \mathrm{~Hz}$ and $10 \mathrm{~Hz}$ in the impedance control, which may be due to the acceleration feedback derived by the twice differentiation from LAE. Therefore, the system must have a wide range of stability in the impedance control with acceleration feedback.

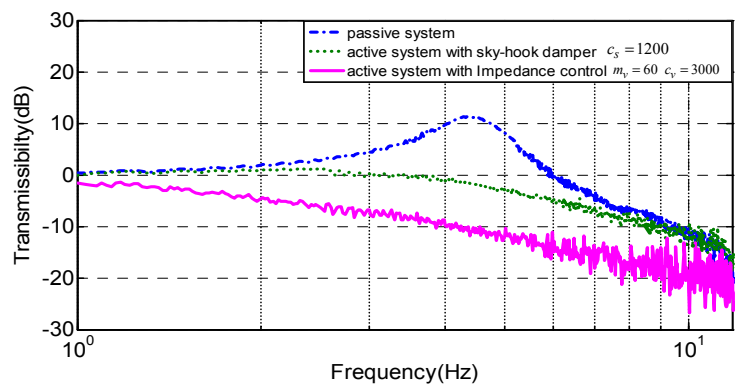

Figure 22. Comparisons of passive system and proposed control structure

\section{Conclusion}

This paper has proposed combining the sky-hook damper and impedance control for an anti-vibration system. The major parameters of the system can be identified by RLS without any supplementary instruments, which could be advantageous for real applications of the proposed anti-vibration system. Further, the natural frequency and damping ratio can be modulated well to actively avoid low frequency vibrations by utilizing the sky-hook damper and impedance control. Not only low frequency vibrations, but also peak resonance can be considered simultaneously. The validity of the proposed approach is confirmed by the experiments.

\section{References}

HWL Scientifice Instrument GmbH. (ND). Active vibration isolation. Retrieved from http://www.tablestable.biz/ pdf/AVI-400-M -web-Di-kompl.pdf

Beard, M. J., Schubert, D. W., \& Von Flotow, A. H. (1994). A practical product implementation of an active/passive vibration isolation system. Proceeding of Active Control of Vibration and Noises, ASME, 75, 485-492. http://dx.doi.org/10.1117/12.188877

Karnopp, D., Crosby, M. J., \& Harwood, R. A. (1974). Vibration control using semi-active force generators. ASME Journal of Engineering for Industry, 96, 619-626. http://dx.doi.org/10.1115/1.3438373

Lee, C. L., Chen, Y. T., Chung, L. L., \& Wang, Y. P. (2006). Optimal design theories and applications of tuned mass dampers. Engineering Structures, 28, 43-53. http://dx.doi.org/10.1016/j.engstruct.2005.06.023 
Lee, S. H., \& Song, J. B. (2001). Acceleration estimator for a low-velocity and low-acceleration regions based on encoder position data. IEEE/ASME Transactions on Mechatronics, 6, 58-64. http://dx.doi.org/10.1109/3516. 914392

Marjoram, R. H. (1985). Pressurized hydraulic mounts for improved isolation of vehicle cabs. SAE Paper \#852349. http://dx.doi.org/10.4271/852349

Tuned mass damper atop the Taipei 101. (ND). Retrieved from http://www.traveljournals.net/pictures/113479.html

Yao, W. S., Hsueh, P. W., \& Tsai, M. C. (2013). Design of an Active-Vibration System Based on Sky-Hook Damper and Impedance Control. Applied Mechanics and Materials, 416-417, 860-865. http://dx.doi.org/10.4028/www.scientific.net/AMM.416-417.860

Yu, Y., Naganathan, N. G., \& Dukkipati, R. V. (2001). A literature review of automotive vehicle engine mounting systems. Journal of Dynamic systems, Mechanism and Machine Theory, 36, 123-142. http://dx.doi.org/10.1016/S0094-114X(00)00023-9

Zhang, H., Ma, R., \& Zhao, D. (2010). Design and parametric study of anti-vibration devices for offshore platforms," Proceedings of International Conference on Computer Engineering and Technology, 5, 6-9. http://dx.doi.org/10.1109/ICCET.2010.5485334

\section{Copyrights}

Copyright for this article is retained by the author(s), with first publication rights granted to the journal.

This is an open-access article distributed under the terms and conditions of the Creative Commons Attribution license (http://creativecommons.org/licenses/by/3.0/). 